\title{
ON APPROXIMATION BY POLYNOMIALS TO A FUNCTION ANALYTIC IN A SIMPLY CONNECTED REGION*
}

\author{
BY O. J. FARRELL
}

In a previous paper $\dagger$ the writer studied expansions in series of polynomials of a function $f(z)$ analytic in a limited simply connected region $G$ where $f(z)$ is known either to be bounded in $G$ or such that the double integral over $G$ of the $p$ th power $(p>0)$ of the modulus of $f(z)$ exists. $\ddagger$ The present note contains an extension of each of the two theorems obtained in the earlier paper. The extended theorems now read as follows.

TheOREM A. Let $G$ be a limited simply connected region of the $z$ plane. Then in order that corresponding to every function $f(z)$ analytic and bounded in $G$ there shall exist a sequence of polynomials $\left\{p_{n}(z)\right\}$ which converge to $f(z)$ in $G$ as $n \rightarrow \infty$ and at the same time such that

$$
\varlimsup_{n \rightarrow \infty}\left[\left|p_{n}(z)\right|, z \text { in } G\right] \leqq \text { bound }[|f(z)|, z \text { in } G]
$$

it is necessary and sufficient that the boundary of $G$ be also the boundary of an infinite region.

THEOREM B. In the a plane let $G$ be a limited simply connected region whose boundary is also the boundary of an infinite region. Let $f(z)$ be analytic in $G$ and such that

$$
\iint_{G}|f(z)|^{p} d S, \quad(p>0),
$$

exists. Then there exists a sequence of polynomials $\left\{p_{n}(z)\right\}$ such that

* Presented to the Society, September 4, 1934.

$\dagger$ This Bulletin, vol. 40 (1934), pp. 908-914.

$\ddagger$ The writer is indebted to Professor J. L. Walsh for having suggested a study of these two problems and also to Professor Torsten Carleman for sending a reprint of his paper on approximation to analytic functions by linear aggregates of prescribed powers (Arkiv för Matematik, Astronomi och Fysik, vol. 17 (1923), pp. 1-30). 


$$
\lim _{n \rightarrow \infty} \iint_{G}\left|f(z)-p_{n}(z)\right|^{p} d S=0
$$

It will be noticed that in the first of these theorems we no longer say that there exist polynomials $\left\{p_{n}(z)\right\}$ which converge to $f(z)$ continuously in $G$, but merely that there exist polynomials $\left\{p_{n}(z)\right\}$ which converge to $f(z)$ in $G$. For if polynomials $\left\{p_{n}(z)\right\}$ converge to $f(z)$ in $G$ so that (1) holds, these polynomials are uniformly bounded in $G$ and thus form in $G$ a normal family of analytic functions from which can be chosen a subsequence converging to $f(z)$ continuously in $G$. Hence, whenever there exists a sequence $\left\{p_{n}(z)\right\}$ converging to $f(z)$ in $G$ so that (1) holds, there exists also a sequence which fulfills (1) and converges to $f(z)$ continuously in $G$. It will be seen too that in the second theorem we no longer say that there exist polynomials $\left\{p_{n}(z)\right\}$ which converge to $f(z)$ continuously in $G$ and for which (3) holds, but merely that there exist polynomials $\left\{p_{n}(z)\right\}$ for which (3) holds. This is because we have since found in the literature a lemma by Walsh* giving assurance that if (3) holds, the polynomials $\left\{p_{n}(z)\right\}$ do converge to $f(z)$ continuously in $G$, so that specific mention of the convergence may be omitted.

The proof of Theorems A and B requires only a slight modification of the proof of the two corresponding theorems in the previous paper. This modification is brought about by observing that if $G$ is any limited simply connected region whose boundary also bounds an infinite region, then there exists a sequence of regions $\left\{G_{n}\right\}$, each of which is a Jordan region lying interior to its predecessor and which are all such that the sequence $\left\{G_{n}\right\}$ converges to $G$ as kernel. $\dagger$ If we use such a sequence of regions $\left\{G_{n}\right\}$, the proofs of Theorems 1 and 2 of the previous paper apply to Theorems $\mathrm{A}$ and $\mathrm{B}$, respectively, of the present note. It is to be remarked, however, that uniform approximation to $f_{n}(z)$ or $F_{n}(z)$ in $\bar{G}$ by a polynomial with arbitrarily small error does not now follow directly from the theorem of Walsh that was 387.

* Transactions of this Society, vol. 33 (1931), pp. 370-388, Lemma on p.

† Compare Carathéodory, Mathematische Annalen, vol. 72 (1912), pp. 107-144, Chapter 3; or Walsh, Transactions of this Society, vol. 32 (1930), pp. 335-390, proof of Theorem X. 
used before, but does follow indirectly from it, since $f_{n}(z)$ or $F_{n}(z)$, being analytic in the closed Jordan region $\bar{G}_{n+1}$, can be uniformly approximated with arbitrarily small error in $\bar{G}_{n+1}$ by a polynomial, and hence can be so approximated in $\bar{G}$. Indeed, Runge's classical theorem on polynomial approximation could be applied here and for that matter could have been used in the previous paper. For the closed region $\bar{G}$ is interior to every region $G_{n}$ and hence the function $f_{n}(z)$ or $F_{n}(z)$ can be approximated as closely as desired in $\bar{G}$ by a polynomial in $z$.

The proof of Theorem B and the proof of the sufficiency of the condition of Theorem A are the same from this point on as for the corresponding theorems in the earlier paper. And the proof of the necessity of the condition of Theorem A is also contained there in $\$ 5$ as "A Remark on Theorem 1."

The writer hopes in a forthcoming paper to be able to determine the most general type of limited simply connected region to which Theorem B can be extended. That this theorem does not hold for an arbitrary finite simply connected region is shown by the following simple example.

Let $G$ be taken as the region bounded by the two circles $|z|=a,|z|=b, b>a$, and by the line segment $a \leqq z \leqq b$. Let $f(z)=1 / z$. Denote by $Q$ the doubly connected region bounded by the two circles. If now there existed a positive number $p$ together with a sequence of polynomials $\left\{p_{n}(z)\right\}$ such that

$$
\lim _{n \rightarrow \infty} \iint_{G}\left|p_{n}(z)-1 / z\right|^{p} d S=0,
$$

it would follow that

$$
\lim _{n \rightarrow \infty} \iint_{Q}\left|p_{n}(z)-1 / z\right|^{p} d S=0 .
$$

Consequently the polynomials $\left\{p_{n}(z)\right\}$ would converge* to $1 / z$ in $Q$ and the convergence would be uniform on any closed point set lying in $Q$, say on a circle $|z|=c, a<c<b$. Hence, the polynomials $\left\{p_{n}(z)\right\}$ would converge uniformly on and within the circle $|z|=c$ to a limit function analytic within this circle. But

* This convergence would follow by the lemma of Walsh to which reference was made above in the paragraph immediately following the statement of Theorem B. 
such a function could not be equal to $1 / z$ in the ring region between the circles $|z|=a$ and $|z|=c$.

The main feature of this example is the use of the lemma of Walsh whereby we know that if in a finite region $R$ we have a sequence of polynomials $\left\{p_{n}(z)\right\}$ for which

$$
\lim _{n \rightarrow \infty} \iint_{R}\left|p_{n}(z)-f(z)\right|^{p} d S=0, \quad(p>0),
$$

where $f(z)$ is a given function analytic in $R$, then the polynomials $\left\{p_{n}(z)\right\}$ converge to $f(z)$ continuously in $R$. The converse is not always true, as was shown by an example in $\$ 4$ of our previous paper. There is, however, a qualified form of converse which does hold for an arbitrary limited region and for an arbitrary function analytic therein. We may state this result as follows.

Theorem C. Let $R$ be a limited region of the z plane, and let $f(z)$ be analytic in $R$ and such that

$$
\iint_{R}|f(z)|^{p} d S, \quad(p>0),
$$

exists. If polynomials $\left\{p_{n}(z)\right\}$ exist which converge to $f(z)$ continuously in $R$ and for which

then

$$
\lim _{n \rightarrow \infty} \iint_{R}\left|p_{n}(z)\right|^{p} d S=\iint_{R}|f(z)|^{p} d S,
$$

$$
\lim _{n \rightarrow \infty} \iint_{R}\left|f(z)-p_{n}(z)\right|^{p} d S=0 .
$$

The proof of this theorem is already contained in the latter part of the proof of Theorem 2 in our previous paper.

We close this note with a result closely connected with Theorem A.

TheOREM D. Let $G$ denote a limited simply connected region whose boundary does not bound an infinite region. Let $B$ denote the boundary of the infinite region among the regions into which the closed region $\bar{G}$ separates the plane, and let $\Gamma$ denote the region (also simply connected) consisting of all the points which can be joined to an arbitrary fixed point of $G$ by a Jordan arc containing 
no point of $B$. Let $f(z)$ be analytic and bounded in $G . A$ necessary and sufficient condition for the existence of polynomials $\left\{p_{n}(z)\right\}$ which converge to $f(z)$ in $G$ so that (1) holds is that there exist a function analytic and bounded in $\Gamma$ and equal to $f(z)$ in $G$.

The proof of this theorem is much the same as for Theorem A taken together with the remark of $\$ 5$ in the earlier paper and is therefore omitted.

The conclusion of Theorem D simply means of course that $f(z)$ shall be analytically extensible throughout $\Gamma$.

Union College

\section{A GENERALIZED PARSEVAL'S RELATION}

BY E. S. QUADE

A function $\phi(x)$ which is non-negative, convex, and satisfies the conditions $\phi(0)=0$ and $(\phi(x) / x) \rightarrow \infty$ as $x \rightarrow \infty$ will be called a Young's function. Given such a function $\phi(x)$, a second function, $\psi(x)$, with the same properties can be found such that Young's inequality, $a b \leqq \phi(a)+\psi(b)$, holds for every $a, b \geqq 0$. The functions $\phi(x)$ and $\psi(x)$ are then said to be complementary in the sense of Young. $\dagger$

If $x(t)$ is such that $\int_{a}^{b} \phi(|x|) d t$ exists, $x(t)$ is said to belong to the space $L_{\phi}(a, b)$. This space is not necessarily linear. $f$ For this reason we denote by $L_{\phi}^{*}(a, b)$ the class of all functions $x(t)$, $a \leqq t \leqq b$, such that the product $x(t) y(t)$ is integrable for every $y(t) \epsilon L_{\psi}(a, b)$. If we put

$$
\|x\|_{\phi}=\sup _{y}\left|\int_{a}^{b} x(t) y(t) d t\right|
$$

for all $y(t)$ with

$$
\rho_{y} \equiv \int_{a}^{b} \psi(|y|) d t \leqq 1,
$$

then $L_{\phi}^{*}$ is a linear metric, and complete space. $\S$ A function

$\dagger$ W. H. Young, Proceedings Royal Society, (A), vol. 87 (1912), pp. 225229.

$\ddagger \mathrm{W}$. Orlicz, Über eine gewisse Klasse von Räumen vom Typus B, Bulletin, Académie Polonaise, (A), Cracovie (1932).

§ A. Zygmund, Trigonometrical Series, 1935, pp. 95-100. 